\title{
FENOLOGÍA DE Unonopsis floribunda Diels y Oxandra sphaerocarpa R.E. Fries (Annonaceae) \\ EN BOSQUES DE LA PLANICIE INUNDABLE DE JENARO HERRERA, LORETO, PERÚ.
}

\section{Luis Freitas ${ }^{1}$ y Lars Peter Kvist ${ }^{2}$}

\section{RESUMEN}

Se presentan y discuten la fenología reproductiva de dos Annonáceas: Unonopsis floribunda Diels y Oxandra sphaerocarpa R.E. Fries. Durante seis años, 34 árboles de Unonopsis floribunda y 15 de Oxandra sphaerocarpa fueron observados en un bosque de la planicie inundable, ubicado a lo largo de la parte baja del río Ucayali en la Amazonía peruana. Todos los árboles crecen en las terrazas naturales, las cuales en la mayoría de los años están inundadas en marzo y abril. Oxandra sphaerocarpa, principalmente florece en el período más seco, de julio a octubre, y fructifica en enero y febrero. La floración de Unonopsis floribunda es bimodal, con picos en junio - julio y en octubre - noviembre, pero varía considerablemente de año en año, aparentemente controlado principalmente por las fluctuaciones del nivel de agua antes que por los patrones de precipitación. Su fructificación es todo el año, pero generalmente con altas frecuencias dos o tres meses después de los picos de floración. Individualmente los árboles tienen frutos por un período extenso, como consecuencia, un alto porcentaje de los árboles usualmente tiene frutos (un promedio de 37 por ciento). Esto corresponde a varios árboles por hectárea en el bosque. La actividad reproductiva está fuertemente correlacionada con el tamaño (DAP) y el crecimiento y menos significativamente con la altura y posición de la copa. Expuestos directamente al sol, ambos, pequeños y grandes árboles, crecen rápidamente y reproducen más frecuentemente.

Palabras claves : Perú, Bosque inundable, Annonaceae, Fenología, Floración, Fructificación.

1 Instituto de Investigaciones de la Amazonía Peruana, Av. Abelardo Quiñonez, Km 2,5, Apartado 784, Iquitos-Perú.

2 Unit of Forestry, Royal Veterinary and Agricultural University, Thorvaldsensvej 57, 1871 Frederiksberg C., Denmark. 


\begin{abstract}
The reproductive phenology of two Annonaceae Unonopsis floribunda Diels and Oxandra sphaerocarpa R.E. Fries are presented and discussed. During six year 34 trees of the former and 15 trees of the latter were observed in a flood plain forest along the lower Ucayali river in the Amazon of Peru. All trees grew at natural levees, which in most years are flooded in march-april. Oxandra sphaerocarpa primarily flowers in the dryest period from july to october, and fruits in january-february. The flowering of Unonopsis floribunda is bimodal with peaks in june-july and in october-november, but the flowering vary considerable from year to year apparently mainly controlled by water level fluctuations rather than precipitation patterns. It fruits the whole year, but generally with the highest frecuencies two to three months after flowering peaks. The individual trees have fruits for an extended period, and a high percentage of the trees usually have fruits (an average of 37 percent). This corresponds to several trees per hectare forest. The reproductive activity is strongly correlated with size (DBH) and growth and less significantly with height and crown position. Exposed to direct sun both smaller and larger trees grow faster and reproduces more frequently.
\end{abstract}

\title{
1. INTRODUCCIÓN
}

En los estudios fenológicos de árboles en la Amazonía desarrollados en bosques no inundables o "tierra firme" (Alencar et al. 1979, Falcao \& Lleras 1979, Gautier \& Spichiger 1986 y Magalhaes \& Alencar 1979), la precipitación parece ser el factor principal que influye en los patrones de reproducción, de modo que la mayoría de especies florecen durante los meses más secos del año, y fructifican al inicio de la estación lluviosa. Las investigaciones sobre fenología de árboles de los bosques de la planicie de inundación son escasas. En éstas, la altura y duración de las inundaciones estacionales son los factores determinantes de la fenología de cada especie, particularmente de aquéllas cuyos frutos y semillas son dispersados por agua y/o peces (Goulding 1979, 1983).

El área de estudio corresponde a una parcela de bosque inundable por agua blanca, rica en sedimentos y nutrientes, en la margen derecha del río Ucayali, en terrazas recientes y fértiles, donde aproximadamente el 10 por ciento de los árboles mayores de $10 \mathrm{~cm}$ de DAP son Annonáceas (Freitas, 1996), siendo las más comunes Unonopsis floribunda y Oxandra sphaerocarpa (Cuadro 1). 
Unonopsis floribunda, es conocida localmente como "Icoja blanca" o "Icoja negra", debido al cambio de coloración del marrón claro suave a oscuro. El extracto de la corteza inmersa en "aguardiente" (licor de caña de azúcar) se usa para el reumatismo y resfríos, y los palos como madera redonda en las construcciones locales.

Oxandra sphaerocarpa comparte el nombre de "Espintana", con especies de Xylopia y también con Guatteria, Malmea y Pseudooxandra. Estas especies proveen aproximadamente el $70 \%$ de la madera redonda usada en construcción en la zona de estudio (Kvist et al. 1995).

\section{2. ÁREA DE ESTUDIO}

El área de estudio está cercana al C.I. Jenaro Herrera, del Instituto de Investigaciones de la Amazonía Peruana (IIAP), distrito de Jenaro Herrera, en el curso inferior del río Ucayali. Las parcelas permanentes se hallan localizadas aproximadamente a 6 kilómetros al sureste de Jenaro Herrera, en el sector "Braga-Supay", donde se prohibe la tala de árboles. El terreno es inundado estacionalmente por agua blanca del río Ucayali, configurado por terrazas naturales de bajiales denominadas "restingas" (Encarnación, 1985, 1993). Los árboles estudiados crecen en terrazas relativamente bajas, entre depresiones más bajas, con una vegetación arbustiva.

La temperatura media anual es de $26,5^{\circ} \mathrm{C}$, con fluctuación mínima durante los meses del año (Claussi et al. 1992, Gautier \& Spichiger 1986), y la precipitación promedio anual de $2687 \mathrm{~mm}$ (Claussi et al. 1992). El período más seco y más soleado (más $200 \mathrm{~h}$ de sol) comprende de junio a setiembre (Figura 1), mientras que en febrero y abril se reduce a $100 \mathrm{~h}$ sol.

La fluctuación anual del nivel de agua en el río Ucayali está basada sobre el promedio del período 1989-1992 (Figura 2). Las máximas crecientes de agua ocurren entre abril y mayo, y el nivel más bajo entre agosto y setiembre. Las principales desviaciones del promedio (Figura 2) se dieron en 1989, cuando el nivel de agua continuó inusualmente alto durante la estación seca y, en 1992, cuando la inundación anual normal fue inusualmente baja, mientras que extraordinariamente creció mucho más en noviembre. En períodos normales el área es inundado desde principios de abril hasta fines de mayo.

Ocasionalmente, no se inunda (por ejemplo en 1992), o el agua alcanza mayor nivel inundando el bosque desde noviembre hasta junio del año siguiente (por ejemplo en 1993 y 1994). 


\section{MÉTODOS}

En 1986, fue demarcado un "arboreto" de 2,8 ha, con un total de 226 árboles seleccionados para los estudios fenológicos, de los que 102 fueron Annonáceas. Las observaciones sistemáticas de estas especies comprendieron desde setiembre de 1986 hasta diciembre de 1994. Para efecto del estudio se consideró el período de seis años de enero de 1987 hasta diciembre de 1992. Los datos de densidad poblacional de las Annonáceas se deducen del análisis de otras tres parcelas, de un total de seis, demarcadas muy cerca del arboreto para estudios de crecimiento y fenología complementarios (Freitas et al, en rev.).

Inicialmente las Annonáceas sólo fueron asignados "in situ” con el nombre vernáculo. En años siguientes se colectaron muestras botánicas de algunos individuos, y 62 de ellas fueron colectadas en noviembre y diciembre de 1994. Las determinaciones de herbario demostraron que los nombres de "Espintana", "Zorro caspi", "Carahuasca" y "Yahuarachi caspi" se usan indistintamente para especies de Guatteria, Malmea, Oxandra, Pseudoxandra y Xylopia, mientras que "Icoja" refiere de modo inequívoco a Unonopsis floribunda.

Las observaciones de los árboles fue con auxilio de binoculares por personal entrenado. Los registros incluyeron la presencia de flores y/o frutos. En 1986 y 1994 se procedió a la medición del DAP; en el último año fueron estimados la altura total, posición y calidad de la copa, y localización topográfica entre las partes más altas y más bajas de las terrazas. Los árboles ubicados en las partes más bajas están expuestos a la inundación por un periodo más largo.

\section{RESULTADOS}

El cuadro 1 presenta a las especies de Annonáceas, de las que Unonopsis floribunda y Oxandra sphaerocarpa fueron estudiadas con 34 y 15 árboles del arborétum respectivamente, cuya densidad corresponde a 13,3 y 13,0 árboles/ha, respectivamente, en las parcelas de estudios estructurales.

El análisis de la fenología para Oxandra sphaerocarpa, en promedio de seis años (figura 3), presenta un único período principal de floración de julio a diciembre, y el pico de fructificación de enero a febrero, antes o durante el periodo de inundación. La variación anual de la fenología no se presenta porque pocos árboles estuvieron fértiles al mismo tiempo. Comparativamente, Unonopsis floribunda en el mismo pe- 
ríodo de seis años, presenta un pico de floración en junio y un segundo de octubre a noviembre; con máxima fructificación a tres meses después de los picos respectivos.

La variación anual de la fenología de Unonopsis floribunda, en los seis años (figura 5), muestra en 1989 un solo pico de floración de setiembre a octubre, en 1990 solamente durante junio, y en 1992 aparece, inusualmente, un tercer pico de enero a febrero. Durante 1992, alrededor del $50 \%$ de los árboles fructificaron por seis meses o más de manera continua. Esta variación imprecisa el comportamiento de fructificación e implica que, en promedio, entre 26 y $46 \%$ de los árboles tienen frutos en cualquier época del año. El porcentaje de árboles en fructificación varía de un mínimo de $9 \%$, en octubre 1989, a un máximo de 88 \% en diciembre de 1992.

El cuadro 2 correlaciona la frecuencia reproductiva de Unonopsis floribunda con un cierto número de parámetros. La correlación más significativa es con el DAP y el crecimiento (en DAP por año), en ambos casos los árboles más grandes y los que están creciendo más rápidamente son progresivamente los más reproductivos. $\mathrm{La}$ altura de los árboles y la posición de la copa son importantes pero menos decisivos, pero los árboles bajos y sombreados (que obviamente a menudo son los mismos) se reproducen con mucho menor frecuencia.

Los árboles establecidos tanto en las restingas como en los bajiales son buenos reproductores, sugiriendo esta observación que la profundidad de la inundación es un factor que no tiene importancia.

El cuadro 3, compara la frecuencia reproductiva de Unonopsis floribunda durante los seis años. La especie tuvo mayor actividad reproductiva durante los dos últimos años, particularmente debido al incremento del tamaño comprobado en las mediciones del DAP de los individuos. Asimismo, se puede observar que los árboles pequeños crecieron, en promedio, más rápidamente que los árboles más grandes.

\section{DISCUSIÓN}

Gautier y Spichiger (1986) y Ríos (1991), investigaron la fenología reproductiva de 34 y 108 especies respectivamente, ubicadas en un arborétum de nueve hectáreas de superficie establecido en tierras altas no inundables, cerca al Centro de Investigaciones Jenaro Herrera. Ellos dividen a los árboles en cuatro categorías fenológicas, y las dos especies investigadas en este trabajo representan dos de estas categorías: Oxandra sphaerocarpa es una especie monomodal de transición, tiene un período 
principal de floración, pero, con algunos individuos floreciendo en otras épocas del año, y Unonopsis floribunda es bimodal, tiene dos picos de floración.

La floración intensiva de Oxandra sphaerocarpa aumentó en forma pronunciada, mientras que el bosque drenaba en mayo y junio, presentando su máximo pico en agosto, cuando el nivel del agua y la precipitación son más bajas (y hay un mayor número de horas con sol) y después decreció correlativamente con el incremento de la precipitación y el nivel del agua (y menos horas de sol) (Figura 1,2,3). Sin embargo, en años normales la intensidad de la floración llega a ser mínima antes que los árboles vuelvan a inundarse en febrero o marzo. Tanto el nivel del agua como las precipitaciones pueden de este modo influenciar la fenología de floración de Oxandra Sphaerocarpa, con el primer parámetro (nivel del agua) se induce la floración y con el segundo (precipitación) se proporciona una entrada para detener la floración.

Durante la mayor parte del año, aproximadamente $10 \%$ de los árboles de Oxandra sphaerocarpa registraron fructificación, con un notable incremento de enero a marzo (Figura 3). La mayoría de los frutos de Oxandra sphaerocarpa parece desarrollar cuando los bosques no están inundados (Figura 2,3), implicando que esta especie no está adaptada a ser dispersada por peces y/o agua. Esto coincide con Van Roosmalen (1985) quien menciona que Oxandra es dispersado por animales (zoochorous).

El primero (en junio) de los dos picos de floración de Unonopsis floribunda (semejante al pico de Oxandra sphaerocarpa) principalmente parece ser afectado por el drenaje del bosque (Figura 2,4,5). Esta posibilidad está apoyada por el hecho que el primer pico fue demorado en 1989 y coincidió con la segunda (Figura 5). En 1989 el nivel del agua permaneció excepcionalmente alto de junio a octubre (aproximadamente $5 \mathrm{~m}$ sobre el promedio de 1989-1992). Después del pico de junio (Figura 4) la intensidad de floración de Unonopsis floribunda disminuye a un mínimo en el mes de agosto, que es el mes seco (excepto en 1989), y luego se incrementa hacia el segundo pico en los meses de octubre a noviembre, que son los más lluviosos pero sin llegar a la inundación (Figuras 1,2,4).

Unonopsis floribunda parece florecer principalmente cuando el bosque no está inundado, pero, si relativamente húmedo. Esto también es sugerido por la dramática disminución de la actividad de la floración de noviembre a diciembre de 1992 (Figura 5), aparentemente provocada por una gran inundación inusualmente anticipada. En contraste, el extraordinario pico de floración en febrero y marzo de 1992 puede reflejar que la inundación a principios de 1992 fue inusualmente baja y tardía (aproximadamente 3 metros debajo del promedio). 
El nivel de agua (o mejor dicho, posiblemente el nivel de agua sobre el terreno) puede influenciar la floración de Unonopsis floribunda más que la cantidad de precipitación (lo cual no se correlaciona con las desviaciones antes mencionadas de la fenología de floración común).

La fenología de fructificación de Unonopsis floribunda no está bien correlacionada con la fluctuación del nivel del río y precipitaciones (Figura 1,2,4,5). Los picos de fructificación se alcanzan generalmente 2-3 meses después de cada pico de floración, al margen de las condiciones que prevalecen durante ese tiempo, por ejemplo, las dos frecuencias de fructificación más altas fueron reportadas en agosto (1991) y diciembre (1992) (Figura 5), cuando el nivel de agua fue el más bajo y el más alto respectivamente.

En cualquier época del año un considerable porcentaje de árboles tiene frutos, siendo el promedio de seis años $37 \%$ (y $20 \%$ con flores). La mayoría de los frutos aparentemente se desarrolla cuando el bosque no está inundado (Figuras 2,4), esto hace suponer que sea más probable que Unonopsis floribunda principalmente sea dispersada por monos y pájaros (endozoochorous), como es mencionado por Van Roosmalen (1985), antes que por los peces y/o agua, tal como es reportado por Gottsberger (1978).

Poblaciones de Unonopsis floribunda pueden proporcionar una abundante y regular fuente de frutos a los dispersadores y/o predadores. Basado en la densidad de la población local de aproximadamente 13 árboles por hectárea de Unonopsis floribunda en el bosque (Cuadro 1), un promedio de más de 4 árboles (37\%) por hectárea pueden tener frutos, con variaciones comunes entre 3 y 6 árboles fructificando, y extremos de 1 a más de 11 (Figura 5). Esta estimación presupone que la población natural es comparable con los árboles seleccionados para observaciones fenológicas; por ejemplo, los valores de DAP son similares, pues, están fuertemente correlacionados desde la actividad reproductiva (Cuadro 2).

Los árboles de Unonopsis floribunda (mayores de $10 \mathrm{~cm}$ ) establecidas en las parcelas permanentes tienen un promedio de DAP de 18,5 cm (1993), mientras que el promedio de DAP de 34 árboles observados fenológicamente creció de 15,5 cm, en 1986, a 20,4 cm en 1994 (aproximadamente $6 \mathrm{~mm}$ por año) (Cuadro 3). Esto indica que, en 1991, el DAP de los 34 árboles observados se incrementó aproximadamente con el mismo promedio que el de las poblaciones naturales de Unonopsis floribunda. En los primeros años del estudio, el DAP en los árboles observados fue menor que en la población local, lo que sugiere que la actividad reproductiva del área de investigación puede haber sido subestimada. 
Las correlaciones positivas entre las frecuencias reproductivas y el DAP, incremento, altura y posición de la copa (Cuadro 2), aparentemente todas reflejan que el principal parámetro causante de la actividad reproductiva de Unonopsis floribunda es la exposición directa a la luz. Sólo los árboles bajo cubierta son significativamente menos reproductivos, y los expuestos individualmente crecen rápidamente y se reproducen al margen de su tamaño.

Individuos expuestos más pequeños (DAP $<10 \mathrm{~cm}$ ) no aplazan la floración hasta que ellos tengan un gran tamaño. El tamaño reproductivo mínimo no puede ser determinado a partir del material de estudio, pues aún los árboles más pequeños observados (que tenían $10 \mathrm{~cm}$ de DAP en 1986) han sido reportados como fértiles. Árboles menores de $10 \mathrm{~cm}$ de DAP pueden consecuentemente sumarse al número de árboles por hectárea en fructificación del bosque, pero producirán un número mínimo de frutos comparado a los árboles grandes.

\section{CONCLUSIÓN}

La fenología de floración de Oxandra sphaerocarpa y Unonopsis floribunda es monomodal de transición y bimodal, respectivamente. Esto parece principalmente estar controlado por las fluctuaciones del nivel de agua, pero los patrones de precipitación (y horas de sol) pueden influenciar particularmente sobre las especies antes mencionadas.

Los frutos se desarrollan a pocos meses después de la floración, al margen del nivel del agua, por ejemplo fructificaciones máximas de Unonopsis floribunda han sido registradas en épocas de vaciantes y en épocas de crecientes grandes. Sólo una pequeña proporción de la producción total de frutos aparentemente se desarrolla durante la inundación, implicando que ninguna de las dos especies están adaptadas para ser dispersadas por los peces o el agua.

Unonopsis floribunda puede proveer un abastecimiento abundante y regular a los frugívoros, puesto que un mínimo de uno y, usualmente, algunos árboles por hectárea del bosque tienen frutos. La fertilidad de Unonopsis floribunda parece principalmente ser promovida por la exposición directa a la luz. Individuos expuestos, grandes y pequeños, crecen rápidamente y usualmente son reproductivos. 


\section{AGRADECIMIENTOS}

Debemos nuestro agradecimiento a los siguientes colaboradores: David Maytahuari y José Valcarcel, quienes condujeron las observaciones fenológicas, Arístides Vásquez y Armando Cáceres, que en 1994 midieron y evaluaron las características silviculturales de los árboles así como por la colección de muestras botánicas. Gustav Nebel, por la preparación de las figuras. Henning Christensen por procesar, ordenar e identificar las muestras botánicas en el Herbario de la Universidad de Aarhus (AAU), Dinamarca, y a Poul Maas, de la Universidad de Utrecht (U), Holanda, por ayudar a identificar el material. A la Agencia Danesa de Desarrollo (Danida) por financiar dos meses de estadía de trabajo a Luis Freitas, en la Real Universidad de Veterinaria y Agricultura (RVAU), Dinamarca, como parte de la colaboración del estudio Bosques Inundables, entre la RVAU, el Instituto de Investigaciones de la Amazonía Peruana (IIAP) y el Departamento de Biología de la Universidad Nacional de la Amazonía Peruana (UNAP). 


\section{BIBLIOGRAFÍA}

ALENCAR, J. DA C.; ALMEIDA, R. A. DE.; FERNÁNDEZ, N. P. 1979. Fenología de espécies florestais em floresta tropical úmida de terra firme na Amazonía Central. Acta Amazonica 9: 163-198.

CLAUSSI, A.; MARMILLOD, D.; BLASER, J. 1992. Descripción silvicultural de las plantaciones forestales de Jenaro Herrera. IIAP, Iquitos, Perú. 334 p.

ENCARNACIÓN, F. 1985. Introducción a la flora y vegetación de la Amazonía Peruana: Estudio actual de los estudios, medio natural y ensayo de una clave de determinación de las formaciones vegetales en la llanura amazónica. Candollea 40: 237-252.

1993. El bosque y las formaciones vegetales en la llanura amazónica del Perú. Alma Máter (Lima, UNMSM) 6: 95-114.

FALCAO, M. DE A.; LLERAS, E. 1980. Aspectos fenológicos, ecológicos e de produtividade do mapati (Pourouma cecropiifolia Mart.). Acta Amazónica 10: 711-724.

FREITAS, L. 1996. Caracterización florística y estructural de cuatro comunidades boscosas de la llanura aluvial inundable en la zona de Jenaro Herrera, Amazonía Peruana. Instituto de Investigaciones de la Amazonía Peruana. Documento Técnico $\mathrm{N}^{\circ} 21.73 \mathrm{p}$.

FREITAS, L.; NEBEL, G.; KVIST, L.P. 1997. Aspectos de ecología y manejo del Capinuri (Maquira coriacea (Karsten) C.C. Berg). Folia Amazónica (en revisión).

GAUTIER, L.; SPICHIGER, R. 1986. Ritmos de reproducción en el estrato arbóreo del Arborétum Jenaro Herrera (provincia de Requena, departamento de Loreto, Perú). Contribución al estudio de la flora y de la vegetación de la Amazonía Peruana, X. Candollea 41: 193-207.

GOTTSBERGER, G. 1978. Seed dispersal by fish in the inundated regions of Humaitá, Amazonía. Biotropica 10: 170-183.

GOULDING, M. 1980. The fishes and the forest: Explorations in Amazonía natural History. Berkely: University of California Press. 280. 
1983. The role of fishes in seed dispersal and plant distribution in Amazonian flood plain Ecosystems. Sonderb. naturwiss. Ver. Hamburg 7: 271-283.

KVIST, L.P.; ANDERSEN, J.K.; HESSELSOE, M.; VANCLAY, J.K. 1995. Estimating use values and relative importance of Amazonian flood plain trees and forests to local inhabitants. Commenwealth Forestry Review 74: 293-300.

MAGALHAES, L.M.S.; ALENCAR, J. DA C. 1979. Fenología do pau-rosa (Aniba duckei Kostermans) en floresta primaria na Amazonía Central. Acta Amazonica 9: 227-232.

ROOSMALEN, M.G.M. VAN. 1985. Annonaceae, pp. 4-21 in Fruits of the Guianan Flora. Utrecht/Wagenigen.

RÍOS, F. 1991. Estudio Fenológico de 108 Especies Forestales del Arborétum Terraza Alta (ATA) Jenaro Herrera-Loreto-Perú. Tesis Ingeniería Forestal. Universidad Nacional de la Amazonía Peruana. 98 p. Iquitos. 
Cuadro1. Densidad en las parcelas de estudios florísticos y número de individuos doservados en el Arboreto, zona de estudio "Braga-Supay".

\begin{tabular}{|l|c|c|}
\hline \multicolumn{1}{|c|}{ Especies } & Parcelas Árb./ha & Arboreto Individuos \\
\hline Duguetia spixiana & 5,3 & - \\
\hline Guatteria sp 1 & 3,3 & 9 \\
\hline Guatteria sp 2 & 1,0 & 6 \\
\hline Malmea sp & 2,3 & 2 \\
\hline Oxandra sphaerocarpa & 13,0 & 15 \\
\hline Pseudooxandra polypheba & 1,3 & 2 \\
\hline Unonopsis floribunda & 13,3 & 34 \\
\hline Xylopia micans & 7,3 & 2 \\
\hline Xylopia sp 1 & 7,0 & - \\
\hline
\end{tabular}

Cuadro2. Influencia del diámetro, crecimiento, altura, posición de la copa y topografía sobre la frecuencia reproductiva de Unonopsis floribunda (1989-1992) .

\begin{tabular}{|c|c|c|c|}
\hline Dap & $<\mathbf{1 8} \mathbf{~ c m}$ & $\mathbf{1 8 - 2 4} \mathbf{~ c m}$ & $>\mathbf{2 4} \mathbf{c m}$ \\
\hline $\mathrm{N}^{\mathbf{o}}$ de árboles & 12 & 12 & 10 \\
\hline Frecuencia & $0,7 / 4,8$ & $0,9 / 5,8$ & $1,3 / 9,2$ \\
\hline Crecimiento (mm por año) & $<\mathbf{5} \mathbf{~ m m}$ & $\mathbf{5 - 9} \mathbf{~ m m}$ & $>\mathbf{~ m m}$ \\
\hline $\mathrm{N}^{\text {o de árboles }}$ & 12 & 13 & 7 \\
\hline Frecuencia & $0,8 / 5,2$ & $0,9 / 5,7$ & $1,3 / 8,8$ \\
\hline Altura & $<\mathbf{1 2} \mathbf{~}$ & $\mathbf{1 2 - 1 8} \mathbf{~ m}$ & $>\mathbf{1 8} \mathbf{~ m}$ \\
\hline $\mathrm{N}^{\text {o de árboles }}$ & 10 & 12 & 10 \\
\hline Frecuencia & $0,6 / 4,2$ & $1,0 / 7,0$ & $1,1 / 7,7$ \\
\hline Posición de la copa & $\mathbf{1 - 2}$ & $\mathbf{3}$ & $\mathbf{4 - 5}$ \\
\hline $\mathrm{N}^{\text {o de árboles }}$ & 11 & 13 & 10 \\
\hline Frecuencia & $0,7 / 4,2$ & $1,0 / 7,0$ & $1,1 / 8,0$ \\
\hline Topografía & Bajo & & Alto \\
\hline $\mathrm{N}^{\text {o de árboles }}$ & 12 & & 22 \\
\hline Frecuencia & $1,0 / 6,8$ & & $0,9 / 6,5$ \\
\hline
\end{tabular}

La frecuencia reproductiva está definida por: 1) El número promedio de sucesos de floración registrados por árbol por año (promedio para todos los árboles 0,9) y, 2). El promedio de meses por año de los árboles que fueron registrados como fértiles (promedio para todos los árboles 6,5). Los parámetros evaluados están correlacionados con la frecuencia reproductiva: DAP (diámetro a la altura del pecho), crecimiento (para los años de 1986 a 1994), altitud, posición de la copa (rango de 1 para los árboles sin ninguna iluminación superior y 5 para los emergentes) y topografía (bajo versus alto en el paisaje). 
FENOLOGÍA DE Unonopsis floribunda Diels y Oxandra sphaerocarpa R.E. Fries (Annonaceae) EN BOSQUES DE LA PLANICIE INUNDABLE DE JENARO HERRERA, LORETO, PERÚ

Cuadro3. Fenología de Unonopsis floribunda ( $\mathrm{n}=32 *)$ durante 1987-1992, en relación con el DAP y número de meses/año.

\begin{tabular}{|c|c|c|c|c|c|c|c|c|c|c|}
\hline \multirow[t]{2}{*}{$\begin{array}{l}\text { DAP } \\
\text { cm. }\end{array}$} & \multirow[t]{2}{*}{$\begin{array}{l}\mathrm{N}^{0} \mathrm{de} \\
\text { Árboles }\end{array}$} & \multicolumn{6}{|c|}{ Años (meses $\left.{ }^{* *}\right)$} & \multicolumn{2}{|c|}{$\begin{array}{c}\text { DAP } \\
\text { Promedio }\end{array}$} & \multirow[t]{2}{*}{$\begin{array}{c}\text { Increm. } \\
\text { Min./año }\end{array}$} \\
\hline & & 87 & 88 & 89 & 90 & 91 & 92 & 86 & 94 & \\
\hline$<13$ & 9 & 4,9 & 5,4 & 4,9 & 4,8 & 9,6 & 7,6 & 11,0 & 16,4 & 6,5 \\
\hline $13-16$ & 13 & 3,8 & $\overline{4,0}$ & 3,9 & 3,1 & 5,8 & $\overline{5,8}$ & 13,5 & 19,5 & 7,2 \\
\hline$>16$ & 10 & 6,5 & 9,9 & 9,5 & 7,3 & 9,2 & 10,7 & 21,1 & 25,3 & 4,1 \\
\hline Total & 34 & 5,0 & 6,4 & 6,1 & 5,3 & 8,2 & 8,3 & 15,5 & 20,4 & 5,9 \\
\hline
\end{tabular}

* Dos árboles no fueron medidos en 1986.

** Refiere a los meses con presencia de flores y/o frutos. 


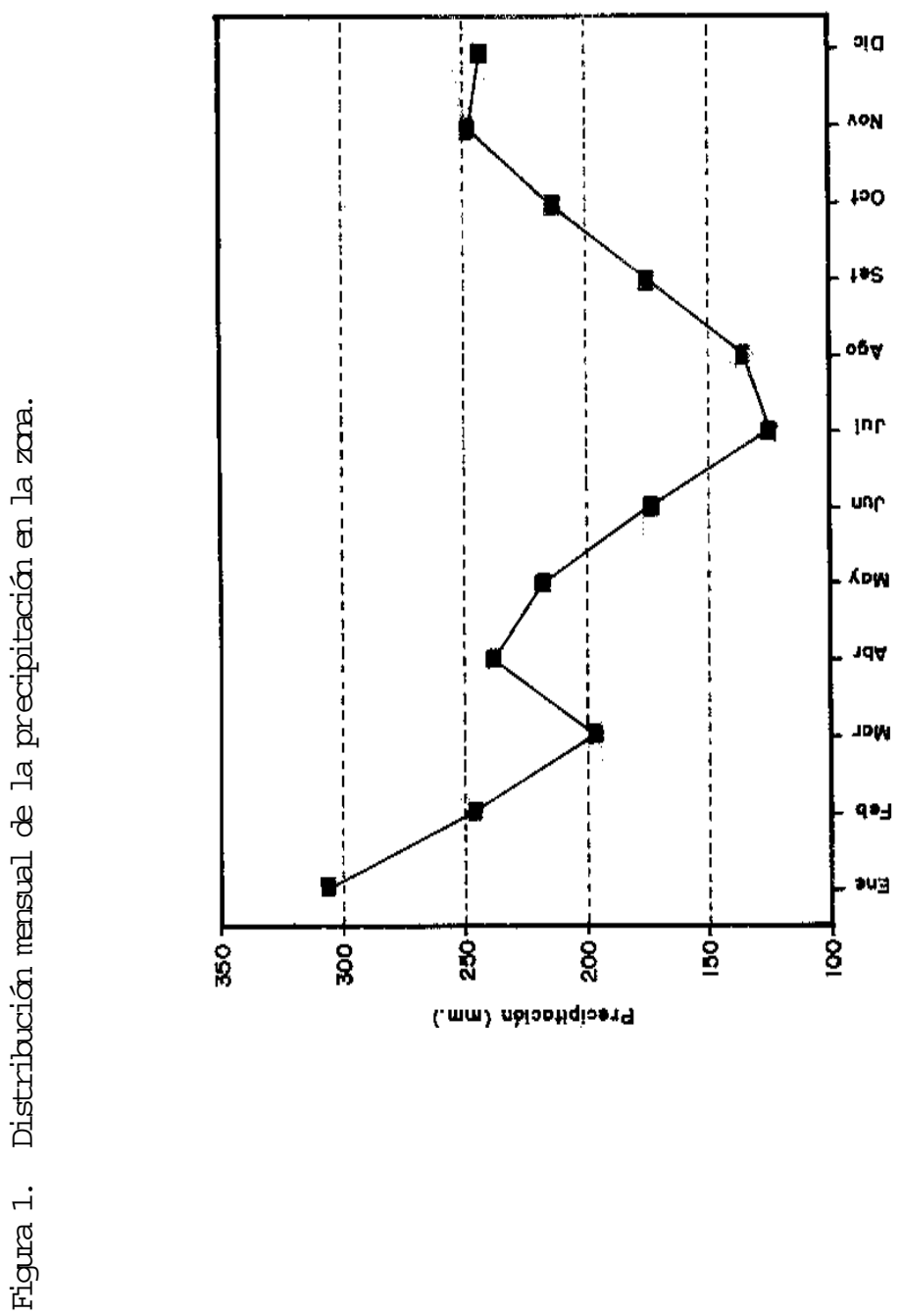


FENOLOGÍA DE Unonopsis floribunda Diels y Oxandra sphaerocarpa R.E. Fries (Annonaceae)

-

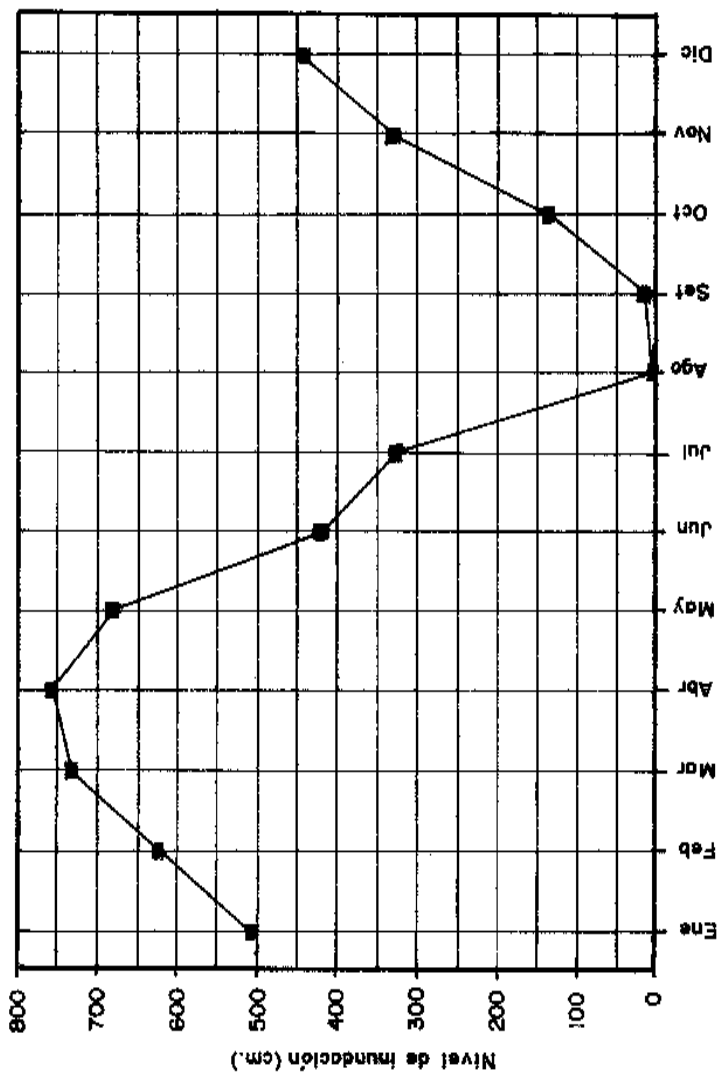



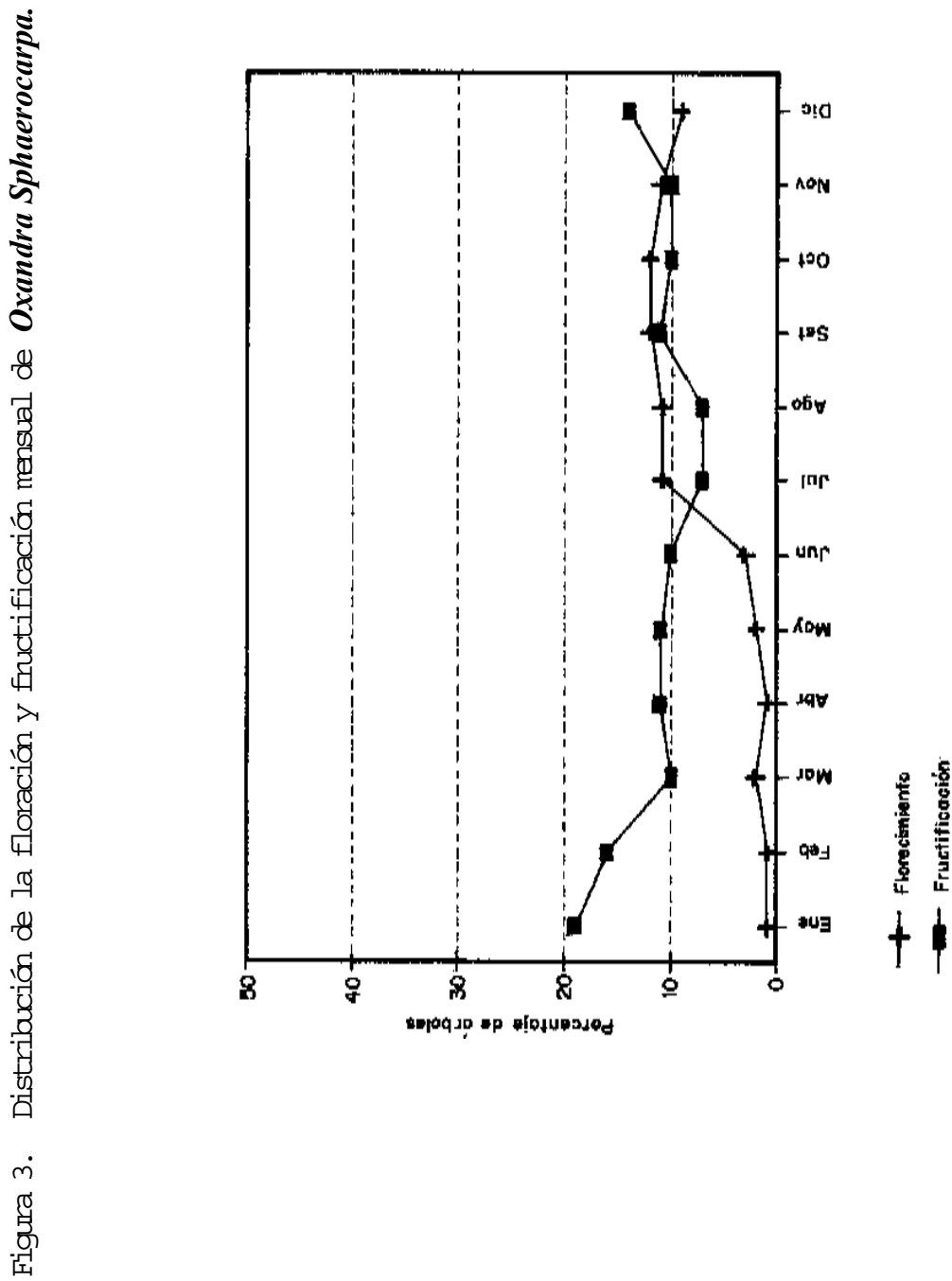
క

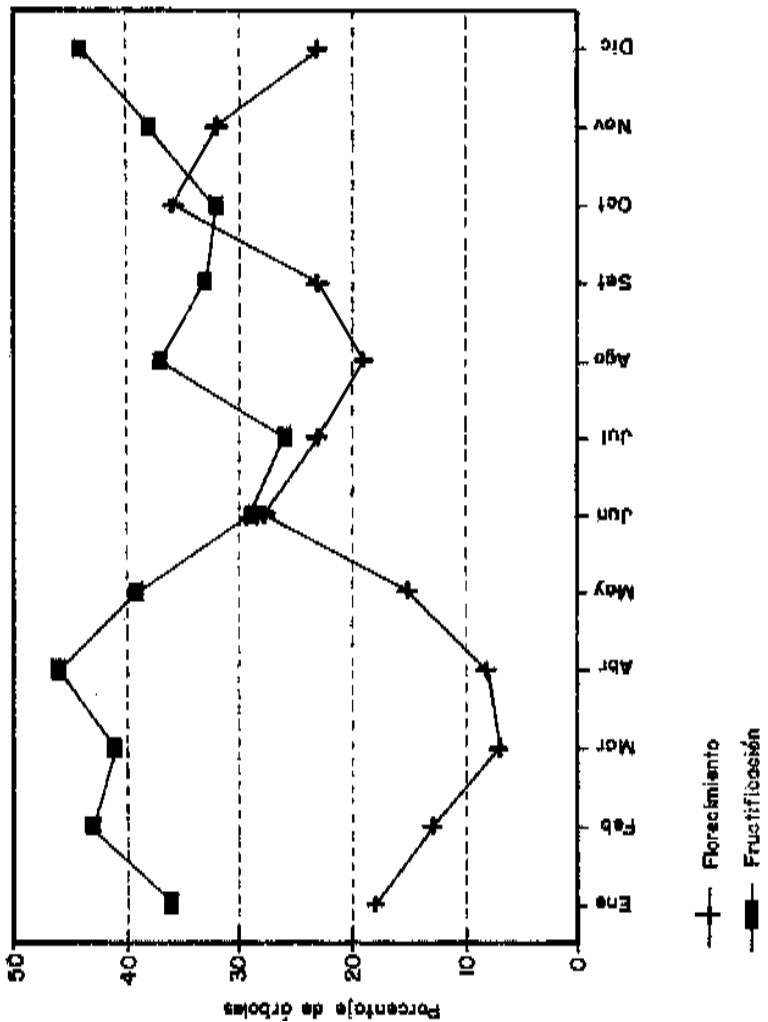


Figura 5. Fenología de Unonopsis floribunda, porcentaje de árboles daservados floreciendo y/o fructificando mensualmente entre 1987 - 1992.
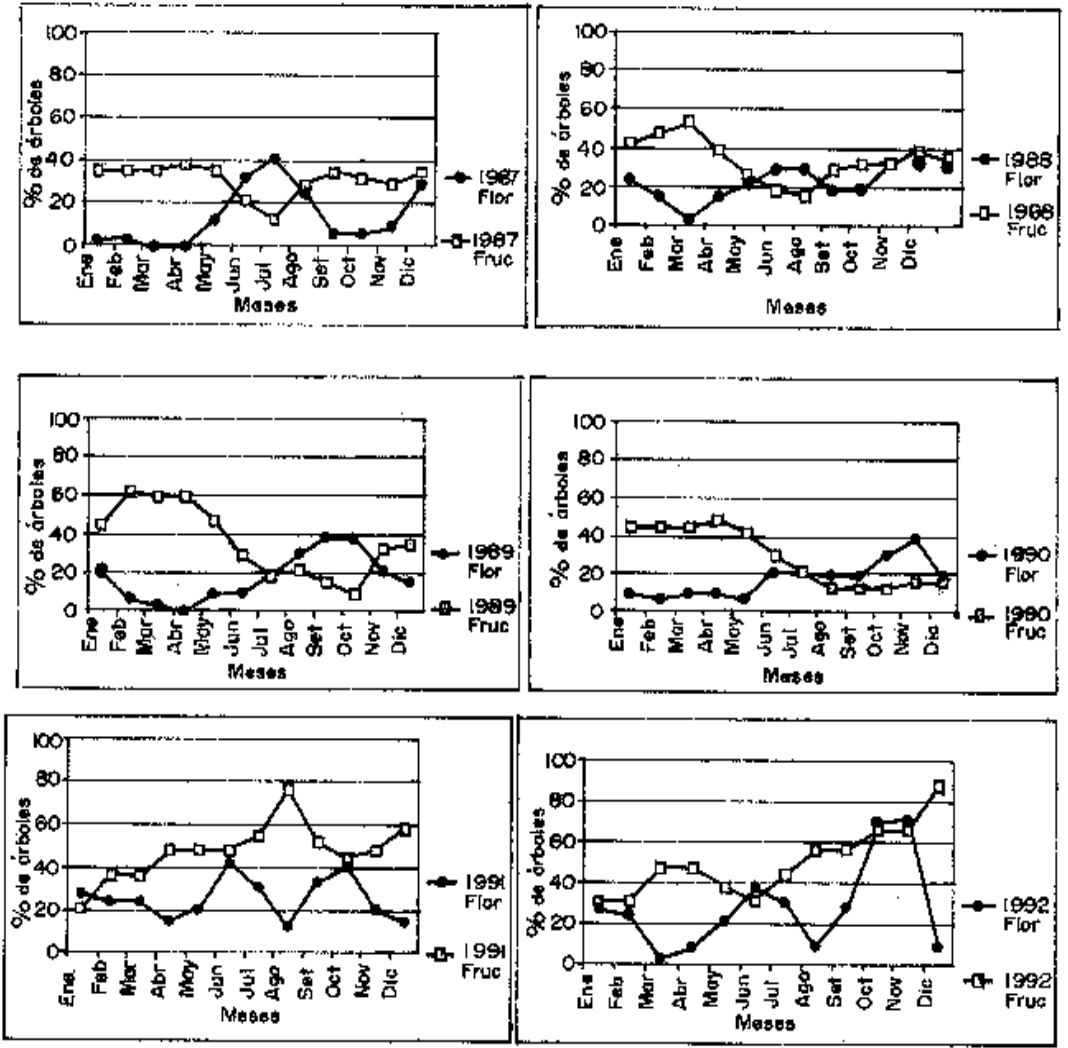